\title{
Analysis of Underwater Disparity Map and Parameter Optimization of SIFT Algorithm
}

\author{
Yongjie Pang ${ }^{1,}$, Di Wang ${ }^{1, b}$ \\ ${ }^{1}$ Science and Technology on Underwater Vehicle Laboratory, Harbin \\ Engineering University, Harbin 150001, China \\ apangyongjie@hrbeu.edu.cn, ${ }^{b}$ andyfighting@163.com
}

\begin{abstract}
.
The depth information of the underwater target is described with the disparity map. So the disparity map generation method is always a hotspot in binocular stereo vision research. The underwater target image stereo matching experiments were carried out by using the BM algorithm, SGBM algorithms and SIFT algorithm in the circulating water channel. Then the advantages and disadvantages of the disparity maps with the three kinds of stereo matching algorithms were analyzed. The analysis results of the disparity maps show that the SIFT stereo matching algorithm was more suitable for underwater stereo matching work. In order to obtain more feature points of underwater images, it is necessary to adjust SIFT algorithm parameter. Underwater image matching experiments were made to determine the appropriate Gaussian kernel parameter $\sigma$. The results illustrated that the improved SIFT algorithm has more feature points with $\sigma=1.9$.
\end{abstract}

Keywords: Stereo Matching; Underwater Disparity Map; Improved SIFT; Gaussian Kernel

\section{Introduction}

Due to the light in the underwater environment is weak, and the visibility is poor, the application of binocular stereo vision technology in the field of underwater robot is a challenging job [1, 2]. 
In order to recover the depth information of the underwater target, it's necessary to generate the dense disparity map based on binocular vision stereo matching. So the disparity map generation method is always a hotspot in binocular stereo vision research [3, 4].

The stereo matching algorithm is the core of the underwater binocular vision technology. At present, the stereo matching methods are commonly used BM method, SGBM method, GC method etc. BM matching algorithm is easy to implement, but it can't optimize the disparity in the whole image [5]. SGBM matching algorithm has strong robustness, but the treatment effect of low texture is not very ideal [6]. Because of large amount of calculation, long operation time, GC algorithm is not suitable for real-time stereo matching work [7]. SIFT algorithm is based on the concept of image characteristic scale. The target object can be matched and identified with the SIFT algorithm in a complex environment $[8,9]$.

In this paper, the BM algorithm, SGBM algorithms and SIFT algorithm were applied to the binocular image matching experiments. Accordingly, the disparity maps were generated. Then the characteristics of the disparity maps were analyzed for the three kinds of stereo matching algorithms. Because the feature points of underwater image are scarce, it is necessary to improved SIFT algorithm parameter. In order to determine the Gaussian kernel parameter $\sigma$, the underwater image matching experiments were carried out by varying Gauss kernel parameter $\sigma$.

\section{The Stereo Matching Method}

BM matching method is a typical matching algorithm based local area. The algorithm uses SAD (Sum of Absolute Difference) to find the matching points between left and right images after three-dimensional rectification.

SGBM semi-global stereo matching algorithm consists of three parts: the pixel-level cost calculation, the cost aggregation, the disparity calculation.

SIFT matching algorithm has better scale, rotation and view angle invariance than the other stereo matching methods. Extreme point is found by comparing with the various scales of feature points. Then the low contrast points and edge response points are eliminated. After generating rotation invariant feature descriptor, the feature points matching can be performed. SIFT matching algorithm consists of five steps:

1) the establishment of scale space 
2) feature points location

3) determing the direction of the feature points

4) the generation of feature points descriptor

5) the feature points matching

\section{Comparative Analysis of the Disparity Maps}

In the circulation water channel $(7 \mathrm{~m} 1.7 \mathrm{~m} 1.5 \mathrm{~m})$, the stereo matching experiments with underwater target were carried out by using the BM algorithm, SGBM algorithms and SIFT algorithm respectively.

First of all, the underwater target images were rectified as is shown in Fig. 1.

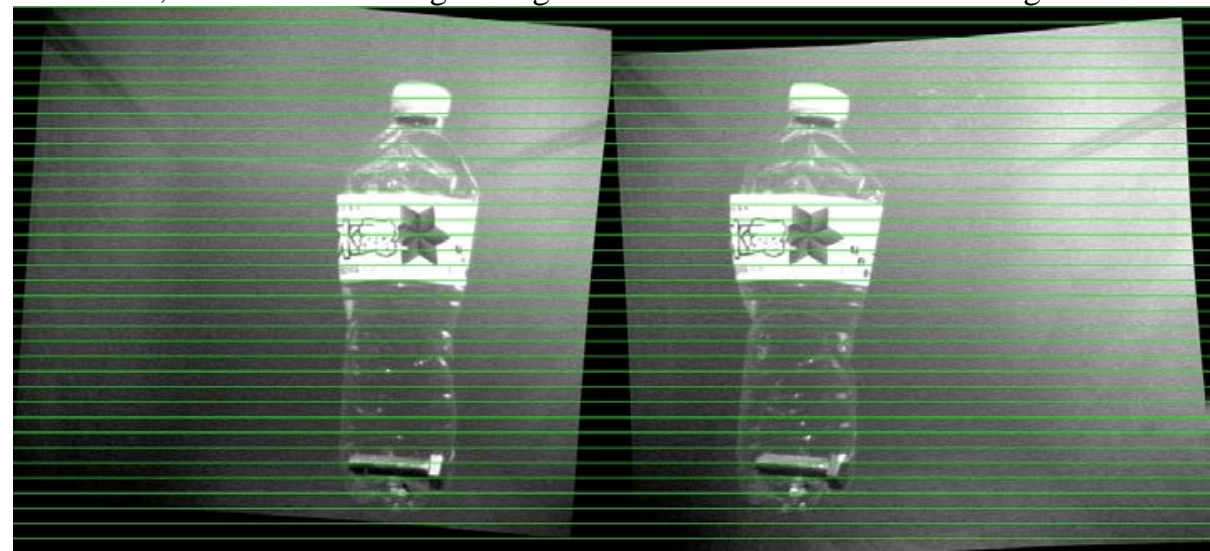

Fig.1 The rectified images

The disparity maps of the bottle using the three algorithms are shown in Fig.2.

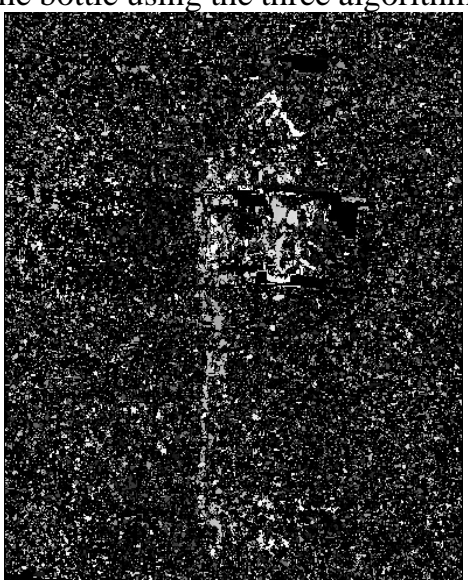

(a) The BM disparity map 


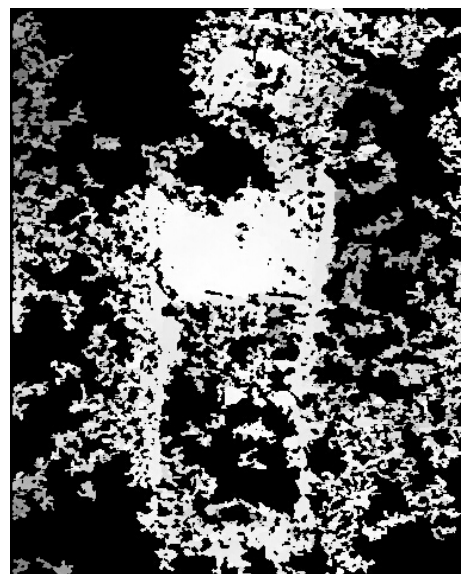

(b) The SGBM disparity map

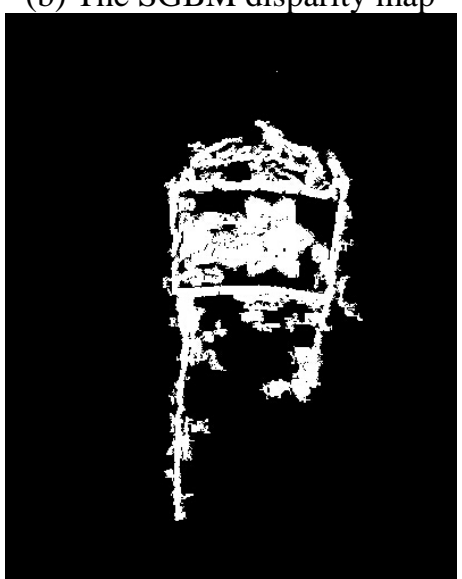

(c) The SIFT disparity map

Fig.2 The disparity maps

In Fig.2a, the matching effect of BM algorithm is poor, although a number of feature points are matched accurately in a certain area. There is a lot of mismatching in the whole image range and the profile of the underwater target is also not clear.

In Fig.2b, the effect of disparity map with SGBM algorithm is better than BM algorithm. But there are still a lot of mismatching feature points in the whole image range.

As is be shown in Fig.2c, the disparity map based on SIFT stereo matching algorithm has a very high accuracy, the feature points of the bottle are displayed precisely. The matching image of the underwater target is smooth and the mismatching points are not obvious. The disparity map with SIFT stereo matching algorithm shows that the final matching results are very close to the true disparity. 
The analysis results illustrated that the SIFT stereo matching algorithm is more suitable for underwater stereo matching work relative to the BM algorithm and SGBM algorithm.

\section{Optimization of SIFT Parameter}

The SIFT algorithm is designed to simulate the multi-scale characteristics of image data. Gauss convolution kernel is the only linear kernel to achieve scale transform. The scale space of a two-dimensional image scale space is defined as:

$$
L(x, y, \sigma)=G(x, y, \sigma) * I(x, y)
$$

$G$ is a variable scale Gauss function.

$$
G(x, y, \sigma)=\frac{1}{2 \pi \sigma^{2}} e^{-\left(x^{2}+y^{2}\right) / 2 \sigma^{2}}
$$

(2)

$\sigma$ is the parameter of the Gauss kernel which determines the smoothness of the image. Through a series of experiments with different images, we found that the choice of parameter $\sigma$ has a significant influence on the number of feature points.

The standard value of Gauss kernel parameter $\sigma$ is 1.6 in the first octave image. The other images are obtained by down sampling.

Because the feature points of underwater image are scarce, it is necessary to improved SIFT algorithm parameter. If the Gauss kernel parameter $\sigma$ is set smaller, the more feature points can be detected in underwater target image.

In order to adjust $\sigma$, the underwater image matching experiments were carried out with the different $\sigma$.And the experimental results are shown in Table 1.

Table 1: The experiment's results with different Gauss kernel parameter $\sigma$

\begin{tabular}{ccc}
$\sigma$ & $\begin{array}{c}\text { The number of feature } \\
\text { points in the left image }\end{array}$ & $\begin{array}{c}\text { The number of feature points } \\
\text { in the right image }\end{array}$ \\
\hline 1.4 & 0 & 0 \\
1.5 & 174 & 164 \\
1.6 & 314 & 321 \\
1.7 & 335 & 365 \\
1.8 & 357 & 388 \\
1.9 & 361 & 413 \\
2.1 & 341 & 396 \\
2.2 & 338 & 387 \\
\hline
\end{tabular}


As shown in Table 1, the number of feature points of the image is not the same with different Gaussian kernel parameter $\sigma$. When $\sigma=1.4$, the feature points of the underwater image can't be detected successfully. With the parameter of the Gauss kernel parameter increases, the number of feature points is gradually increasing in the underwater target image. But the number of feature points is beginning to show a downward trend when the Gaussian kernel parameter $\sigma$ increases to a certain extent. As shown in Table 1, the number of feature points started to decrease with $\sigma=2.1$.

In conventional SIFT algorithm, the parameter $\sigma$ is set to 1.6. For the underwater image, the number of feature points is insufficient with $\sigma=1.6$.

In Table 1, when $\sigma=1.9$, the number of feature points of the underwater image reaches the maximum, which provide a good condition for the subsequent stereo matching.

The disparity maps of the underwater target were generated with the $\sigma=1.6$, $\sigma=1.9$, as is shown in Fig. 3

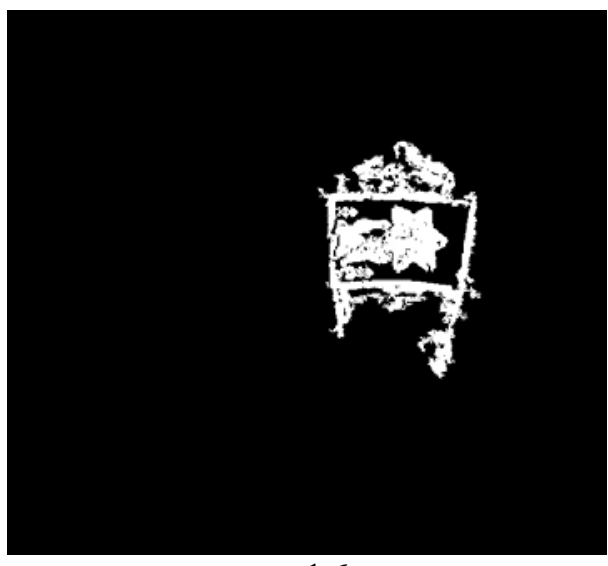

$\sigma=1.6$ 


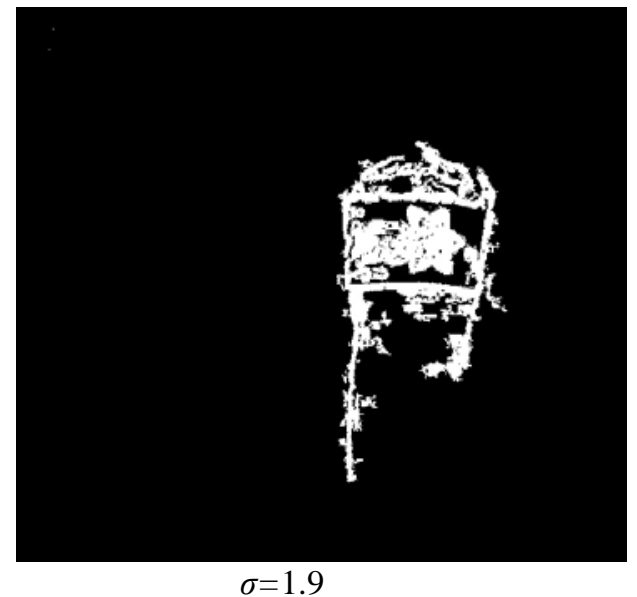

Fig.3 Disparity maps with the different $\sigma$

Compared to $\sigma=1.6$, the underwater disparity map generates more match points, and the profile of the target is clearer with $\sigma=1.9$. When $\sigma=1.9$, the SIFT algorithm has been improved, which is more suitable for the generation of underwater disparity map.

\section{Conclusion}

Compared with the BM algorithm and SGBM algorithms, the SIFT algorithm has been proved to be more suitable for underwater stereo matching. In order to determine the more suitable Gaussian kernel parameter $\sigma$, several underwater image matching experiments were carried out by varying Gauss kernel parameter $\sigma$. The results illustrated that the improved $\sigma$ is better than the standard value for underwater disparity map generation.

\section{Acknowledgements}

This work was financially supported by the National Science Foundation of China (51209050), the Doctoral Fund of Ministry of Education for Young Scholar (20122304120003) and State Key Laboratory of Robotics and Systems of Harbin Institute of Technology (SKLRS-2012-ZD-03).

\section{References}

[1] J. Aloimonos, L. Weiss and A. Bandopadhay: Internation Journal of Computer Vision Vol. 1-4 (2008), p. 302 
[2] J.R. Neves, A.J. Pinho and D.A. Martins: Mechatronics Vol. 21-2 (2011), p. 119

[3] Jilong. Bian, Chaoguang. Men and Xiang. Li: Journal of Electronics \& Information Technology Vol. 34-3 (2012), p. 517

[4] E. Menegatti, A. Pretto and A. Scarpa: IEEE Transactions on Robotics Vol. 22-3 (2006), p. 523

[5] G. Bradski and A. Kaehler: Learning OpenCV (O'Reilly Media Press, 2008).

[6] L.M. Surhone, M.T. Tennoe and S.F. Henssonow: OpenCV: computer vision, intel corporation, willow garage (Flu Press, 2011).

[7] Daolei. Wang and K.B. Lim: Journal of Visual Communication and Image Repressentation Vol. 22-4 (2011), p. 325

[8] D.G. Lowe: International Journal of Computer Vision Vol. 60-2 (2004), p. 91

[9] C. Beyan and A. Temizel: IET Computer Vision Vol. 6-1 (2012), p. 12 